\title{
Revisiting simplified dark matter models in terms of AMS-02 and Fermi-LAT
}

\author{
Tong Li \\ School of Physics, Nankai University, \\ Tianjin 3000\%1, China \\ ARC Centre of Excellence for Particle Physics at the Tera-scale, \\ School of Physics and Astronomy, Monash University, \\ Melbourne, Victoria 3800, Australia \\ E-mail: nklitong@hotmail.com
}

ABSTRACT: We perform an analysis of the simplified dark matter models in the light of cosmic ray observables by AMS-02 and Fermi-LAT. We assume fermion, scalar or vector dark matter particle with a leptophobic spin-0 mediator that couples only to Standard Model quarks and dark matter via scalar and/or pseudo-scalar bilinear. The propagation and injection parameters of cosmic rays are determined by the observed fluxes of nuclei from AMS-02. We find that the AMS-02 observations are consistent with the dark matter framework within the uncertainties. The AMS-02 antiproton data prefer $30(50) \mathrm{GeV}-5 \mathrm{TeV}$ dark matter mass and require an effective annihilation cross section in the region of $4 \times 10^{-27}\left(7 \times 10^{-27}\right)-4 \times 10^{-24} \mathrm{~cm}^{3} / \mathrm{s}$ for the simplified fermion (scalar and vector) dark matter models. The cross sections below $2 \times 10^{-26} \mathrm{~cm}^{3} / \mathrm{s}$ can evade the constraint from Fermi-LAT dwarf galaxies for about $100 \mathrm{GeV}$ dark matter mass.

Keywords: Beyond Standard Model, Cosmology of Theories beyond the SM

ARXIV EPRINT: 1708.04534 


\section{Contents}

1 Introduction 1

2 The simplified dark matter models 3

3 Indirect observables from AMS-02 and Fermi-LAT 4

3.1 Antiproton flux from AMS-02 4

$\begin{array}{ll}3.2 \text { Dwarf galaxy constraint from Fermi-LAT } & 7\end{array}$

$\begin{array}{llr}4 & \text { Results } & 8\end{array}$

5 Conclusions 14

A Expressions of mediator decay widths and dark matter annihilation cross sections

A.1 fermion dark matter model $D_{2}$

$\begin{array}{lll}\text { A.2 fermion dark matter model } D_{4} & 15\end{array}$

$\begin{array}{lll}\text { A.3 scalar dark matter model } C & 15\end{array}$

$\begin{array}{ll}\text { A.4 vector dark matter model } V & 16\end{array}$

\section{Introduction}

The measurements of Galactic cosmic rays provide crucial information to understand their own source and propagation and further confine the possibly new fundamental particle physics such as dark matter (DM) annihilation. Cosmic rays in the Galaxy are categorized into primary and secondary types according to their different origins [1-4]. Primary cosmic rays are those which are created by astrophysical sources, while their initial spectrum and composition change and thus emit secondary cosmic rays as a result of interacting with matter in the interstellar medium (ISM). The secondary-to-primary ratio of cosmic ray nuclei (such as the Boron-to-Carbon ratio B/C) and the ratio of secondary isotopes (such as the Beryllium ratio ${ }^{10} \mathrm{Be} /{ }^{9} \mathrm{Be}$ ) are widely employed to constrain the cosmic ray propagation parameters as they are respectively sensitive to the traveling path and the lifetime of cosmic rays in the Galaxy. The source injection parameters of cosmic ray nuclei can be constrained by the measured proton flux data.

Recently, AMS-02 collaboration released abundant and precise data on the cosmic ray nuclei, e.g. proton [5], B/C [6], etc. Combining with old data sets of ${ }^{10} \mathrm{Be} /{ }^{9} \mathrm{Be}$ from ACE [7] and proton from PAMELA [8], one can constrain the propagation and source injection parameters in a statistical method. Based on these parameters, an up-to-date antiproton cosmic rays as the secondary production from colliding protons with ISM can be obtained with high precision. Given this astrophysical background of antiproton, we are enabled to confine extra compositions in cosmic rays such as annihilating dark matter 
which also produces antiproton, in the light of the antiproton flux data newly reported by AMS-02 [9].

In addition to charged cosmic rays, the gamma ray flux is also an observable that dark matter can produce potentially. Dwarf galaxies are one of the best places with a large abundance of dark matter and thus the bright targets to search for gamma rays from dark matter annihilation. The Fermi Large Area Telescope (LAT) has looked for gamma ray emission from the dwarf spheroidal satellite galaxies (dSphs) of the Milky Way and detected no excess. Fermi-LAT thus placed upper limit on the dark matter annihilation cross section from a combined analysis of 15 Milky Way dSphs [10] and recently updated the result with more candidate dSphs and increased sensitivity [11]. They are generally most stringent constraints for dark matter annihilating into quark or gluon channels [12].

In this work, we examine the constraints set by the AMS-02 antiproton data and the Fermi-LAT dSphs on the simplified models with weakly interacting mass particle (WIMP) as dark matter. Specifically we consider Dirac fermion, complex scalar and vector dark matter denoted by $\chi, \phi$ and $X$ respectively, with mediators that only couple to the Standard Model (SM) quarks and dark matter particles. This leptophobic framework is widely used to analyze the data for dark matter search in indirect detection (ID) and direct detection (DD) and collider experiments [13-20]. It uses minimal and general theoretical assumptions with only two parameters, i.e. the dark matter mass and the mediator mass.

In the simplified framework the annihilation of dark matter in s-channel occurs through the exchange of either a spin- 0 or spin- 1 mediator. The general interactions between the mediator and dark matter or SM quarks are the Lorentz-invariant combinations of the following bilinears

$$
\begin{aligned}
& \bar{\chi} \chi, \bar{\chi} \gamma^{5} \chi, \bar{\chi} \gamma^{\mu} \chi \text { (Dirac only), } \bar{\chi} \gamma^{\mu} \gamma^{5} \chi ; \phi^{\dagger} \phi, \phi^{\dagger} \overleftrightarrow{\partial_{\mu}} \phi \text { (complex only); } X_{\mu}^{\dagger} X^{\mu}, X_{\mu}^{\dagger} \partial_{\nu} X^{\nu} \\
& m_{q} \bar{q} q, m_{q} \bar{q} \gamma^{5} q, \bar{q} \gamma^{\mu} q, \bar{q} \gamma^{\mu} \gamma^{5} q .
\end{aligned}
$$

As dictated by minimal flavor violation, the couplings of scalar and pseudo-scalar quark bilinears are scaled by quark mass $m_{q}$ and those of vector and axial-vector bilinears are chosen to be universal $[21,22]$. The spin-1 mediator scenario via vector or axial-vector interaction is thus highly constrained by the dijet limit for $Z^{\prime}$ search at the Large Hadron Collider (LHC) [23-25]. The collider search is yet less sensitive to the detection of spin-0 mediator scenario through scalar or pseudo-scalar interaction as a result of the $m_{q}^{2}$ suppression coming from the Yukawa coupling. Among the structure combinations in the spin-0 mediator scenario only four forms lead to annihilation cross section without velocity suppression, denoted by $D_{2}, D_{4}, C$ and $V$ using the notation of effective field theories (EFTs) [22], as shown in table 1. Moreover, for these models, the nucleon-WIMP scattering rates are either suppressed by the spin of the target nucleus and/or dark matter and the scattering momentum exchange [26], rendering weak DD constraints. We thus investigate the sensitivity of AMS-02 and Fermi-LAT indirect observables to the detection of simplified dark matter models $D_{2}, D_{4}, C$ and $V$.

This paper is organized as follows. In section 2, we discuss the simplified dark matter models we use. In section 3 we describe the observales from AMS-02 and Fermi-LAT. Our numerical results are given in section 4 . Finally, in section 5 we summarize our conclusions. 


\begin{tabular}{|c|c|c|c|}
\hline Interations & ID & DD & Collider \\
\hline $\mathrm{D}_{2}: \bar{\chi} \gamma_{5} \chi \oplus \bar{q} q$ & 1 & $\mathbf{s}_{\chi} \cdot \mathbf{q}$ & $m_{q}^{2}$ \\
\hline $\mathrm{D}_{4}: \bar{\chi} \gamma_{5} \chi \oplus \bar{q} \gamma_{5} q$ & 1 & $\left(\mathbf{s}_{\chi} \cdot \mathbf{q}\right)\left(\mathbf{s}_{N} \cdot \mathbf{q}\right)$ & $m_{q}^{2}$ \\
\hline $\mathrm{C}: \phi^{\dagger} \phi \oplus \bar{q} \gamma_{5} q$ & 1 & $\mathbf{s}_{N} \cdot \mathbf{q}$ & $m_{q}^{2}$ \\
\hline $\mathrm{V}: X_{\mu}^{\dagger} X^{\mu} \oplus \bar{q} \gamma_{5} q$ & 1 & $\mathbf{s}_{N} \cdot \mathbf{q}$ & $m_{q}^{2}$ \\
\hline
\end{tabular}

Table 1. Interactions considered in this work and their suppression effects for ID, DD and collider search. $\mathbf{s}_{\chi}\left(\mathbf{s}_{N}\right)$ is the spin of dark matter (the target nucleus) and $\mathbf{q}$ is the scattering momentum exchange.

\section{The simplified dark matter models}

In this section, we describe the considered simplified dark matter models in table 1 . The dark matter particles $(\chi, \phi, X)$ couple to the SM quarks through a spin-0 mediator $S_{2}$, $S_{4}, S_{C}$ or $S_{V}$ corresponding to structure $D_{2}, D_{4}, C$ or $V$ respectively. The corresponding interactions are as follows $[16,27]$

$$
\begin{aligned}
\mathcal{L}_{\mathrm{D} 2} & =-i g_{\chi}^{\mathrm{D} 2} S_{2} \bar{\chi} \gamma_{5} \chi-S_{2} \sum_{q=u, d, s, c, b, t} g_{q}^{\mathrm{D} 2} \frac{m_{q}}{v_{0}} \bar{q} q, \\
\mathcal{L}_{\mathrm{D} 4} & =-i g_{\chi}^{\mathrm{D} 4} S_{4} \bar{\chi} \gamma_{5} \chi-i S_{4} \sum_{q=u, d, s, c, b, t} g_{q}^{\mathrm{D} 4} \frac{m_{q}}{v_{0}} \bar{q} \gamma_{5} q, \\
\mathcal{L}_{\mathrm{C}} & =-g_{\phi}^{\mathrm{C}} m_{\phi} S_{C} \phi^{\dagger} \phi-i S_{C} \sum_{q=u, d, s, c, b, t} g_{q}^{\mathrm{C}} \frac{m_{q}}{v_{0}} \bar{q} \gamma_{5} q, \\
\mathcal{L}_{\mathrm{V}} & =-g_{X}^{\mathrm{V}} m_{X} S_{V} X_{\mu}^{\dagger} X^{\mu}-i S_{V} \sum_{q=u, d, s, c, b, t} g_{q}^{\mathrm{V}} \frac{m_{q}}{v_{0}} \bar{q} \gamma_{5} q,
\end{aligned}
$$

where $v_{0}=246 \mathrm{GeV}$ is the Higgs vacuum expectation value. Following the general choices in the analysis of dark matter searches in literatures, we take $g_{\chi}^{\mathrm{D} 2}=g_{\chi}^{\mathrm{D} 4}=g_{q}^{\mathrm{D} 2}=g_{q}^{\mathrm{D} 4}=g_{\phi}^{\mathrm{C}}=$ $g_{X}^{\mathrm{V}}=g_{q}^{\mathrm{C}}=g_{q}^{\mathrm{V}}=1$ in the calculations below. Under the above assumptions the dark matter models are described by two parameters, i.e. the dark matter mass $m_{\mathrm{DM}}=m_{\chi}, m_{\phi}, m_{X}$ and the mediator mass $m_{\mathrm{Med}}=m_{S_{2}}, m_{S_{4}}, m_{S_{C}}$ or $m_{S_{V}}$. We scan these parameters in the following range

$$
5 \mathrm{GeV}<m_{\mathrm{DM}}, m_{\mathrm{Med}}<10 \mathrm{TeV} .
$$

Given the interactions in eqs. (2.1) and (2.2), the pairs of dark matter particle can either annihilate into SM quark or gluon pairs via $\mathrm{DM} \overline{\mathrm{DM}} \rightarrow \mathrm{Med} \rightarrow \bar{q} q, g g(\mathrm{DM}=\chi, \phi, X$; Med $\left.=S_{2}, S_{4}, S_{C}, S_{V}\right)$ or annihilate into four SM quarks or gluons via two mediators in t-channel DM DM $\rightarrow$ Med Med $\rightarrow 4$ quarks, 4 gluons when kinematically allowed. The energy distribution of cosmic rays produced in the annihilation, as a result, is the sum of 2-body spectrum and 4-body spectrum

$$
d N_{i} / d E=\left(d N_{i} / d E\right)_{2-\text { body }}+\left(d N_{i} / d E\right)_{4-\text { body }},
$$

where $i$ denotes the cosmic ray species, i.e. $\bar{p}, \gamma$ here. The two types of spectrum are both the annihilation-fraction-weighted sum of the differential yields into specific final states. For 
different quark or gluon final states in 2-body spectrum we use PPPC4DMID [28] to generate the differential yield which is weighted by the corresponding annihilation fraction, i.e. $\left\langle\sigma_{\text {ann }} v\right\rangle_{\bar{q} q} /\left\langle\sigma_{\text {ann }} v\right\rangle$ and $\left\langle\sigma_{\text {ann }} v\right\rangle_{g g} /\left\langle\sigma_{\text {ann }} v\right\rangle$. The 4 -body cosmic ray spectrum is given by the spectrum of the mediator decay in its rest frame followed by a Lorentz boost $[12,29]$. The spectrum is then weighted by the product of annihilation fraction and decay branching ratio of the mediator, i.e. $\frac{\left\langle\sigma_{\text {ann }} v\right\rangle_{\text {Med }}}{\left\langle\sigma_{\text {ann }} v\right\rangle} \frac{\Gamma_{\text {Med } \rightarrow q \bar{q}}}{\Gamma_{\text {Med }}}$ and $\frac{\left\langle\sigma_{\text {ann }} v\right\rangle_{\text {Med }}}{\left\langle\sigma_{\text {ann }} v\right\rangle} \frac{\Gamma_{\text {Med } \rightarrow g g}}{\Gamma_{\text {Med }}}$, to give $\left(d N_{i} / d E\right)_{4-\text { body }}$ [30]. The expressions of mediator decay widths and dark matter annihilation cross sections are collected in appendix.

\section{Indirect observables from AMS-02 and Fermi-LAT}

In this section we describe the observables of antiproton flux and gamma ray measured by AMS-02 and Fermi-LAT respectively.

\subsection{Antiproton flux from AMS-02}

The two key unknowns about cosmic rays in the Galaxy are their origin and propagation. The propagation of cosmic rays can be described as the process of diffusion. The diffusion process is written in the form of the transport equation below [31]

$$
\begin{aligned}
\frac{\partial \psi}{\partial t}= & Q(\vec{r}, p)+\vec{\nabla} \cdot\left(D_{x x} \vec{\nabla} \psi-\vec{V} \psi\right)+\frac{\partial}{\partial p} p^{2} D_{p p} \frac{\partial}{\partial p} \frac{1}{p^{2}} \psi \\
& -\frac{\partial}{\partial p}\left[\dot{p} \psi-\frac{p}{3}(\vec{\nabla} \cdot \vec{V}) \psi\right]-\frac{\psi}{\tau_{f}}-\frac{\psi}{\tau_{r}}
\end{aligned}
$$

where $\psi(\vec{r}, t, p)$ is the density of cosmic rays, $\vec{V}$ is the convection velocity and $\tau_{f}\left(\tau_{r}\right)$ is the time scale for fragmentation (radioactive decay). $\dot{p}$ is the momentum loss rate. The convection terms in the above equation are induced by the Galactic wind. The diffusion in momentum space governs the reacceleration process. In this case the diffusion coefficient in momentum space, i.e. $D_{p p}$, is related to the spatial coefficient $D_{x x}$ and the Alfven velocity $v_{A}[32]:$

$$
D_{p p} D_{x x}=\frac{4 p^{2} v_{A}^{2}}{3 \delta\left(4-\delta^{2}\right)(4-\delta) w},
$$

with the level of the interstellar turbulence parameter $w$ being 1 . The spatial diffusion coefficient is usually written in this form

$$
D_{x x}=\beta D_{0}\left(R / R_{0}\right)^{\delta},
$$

with $R$ and $\beta$ being the rigidity and particle velocity divided by light speed respectively. This transport equation is numerically solved based on given boundary conditions, that is, the cosmic ray density $\psi$ vanishes at the radius $R_{h}$ and the height $z_{0}$ of the cylindrical diffusion halo.

In eq. (3.1), the source term can be written by the product of the spatial distribution and the injection spectrum function for cosmic ray species $i$

$$
Q_{i}(\vec{r}, p)=f(r, z) q_{i}(p) \text {. }
$$




\begin{tabular}{|c|c||c|c||c|c|}
\hline propagation & value & nucleon injection & value & solar modulation & value \\
\hline$D_{0}\left(10^{28} \mathrm{~cm}^{2} \mathrm{~s}^{-1}\right)$ & 7.24 & $\nu_{1}$ & 1.69 & $\phi_{p}(\mathrm{MV})$ & 550 \\
\hline$\delta$ & 0.38 & $\nu_{2}$ & 2.37 & $\phi_{\bar{p}}(\mathrm{MV})$ & 400 \\
\hline$R_{0}(\mathrm{GV})$ & 4 & $R_{\mathrm{br}}^{p}(\mathrm{GV})$ & 12.88 & - & - \\
\hline$v_{A}\left(\mathrm{~km} \mathrm{~s}^{-1}\right)$ & 38.5 & $A_{p}($ see caption $)$ & 4.498 & - & - \\
\hline$z_{0}(\mathrm{kpc})$ & 5.93 & - & - & - & - \\
\hline
\end{tabular}

Table 2. Parameters of propagation, nucleon injection and solar modulation and their values adopted in our numerical analysis. The proton flux is normalized to $A_{p}$ at $100 \mathrm{GeV}$ in the units of $10^{-9} \mathrm{~cm}^{-2} \mathrm{~s}^{-1} \mathrm{sr}^{-1} \mathrm{MeV}^{-1}$.

We use the following supernova remnants distribution for the spatial distribution of the primary cosmic rays

$$
f(r, z)=f_{0}\left(\frac{r}{r_{\odot}}\right)^{a} \exp \left(-b \frac{r-r_{\odot}}{r_{\odot}}\right) \exp \left(-\frac{|z|}{z_{s}}\right)
$$

where $r_{\odot}=8.5 \mathrm{kpc}$ is the distance between the Sun and the Galactic center [33], the height of the Galactic disk is $z_{s}=0.2 \mathrm{kpc}$. The two parameters $a$ and $b$ are chosen to be 1.25 and 3.56 , respectively [34]. The following power law with one break is assumed for the injection spectrum of various nuclei

$$
q_{i} \propto\left\{\begin{array}{l}
\left(R / R_{\mathrm{br}}^{p}\right)^{-\nu_{1}}, R \leq R_{\mathrm{br}}^{p} \\
\left(R / R_{\mathrm{br}}^{p}\right)^{-\nu_{2}}, R>R_{\mathrm{br}}^{p}
\end{array}\right.
$$

where the rigidity break $R_{\mathrm{br}}^{p}$ and power law indexes $\nu_{1}, \nu_{2}$ are injection parameters.

The above propagation parameters and source injection parameters can be constrained by fitting the ratios of nuclei, i.e. the Boron-to-Carbon ratio $(\mathrm{B} / \mathrm{C})$ and the Beryllium ratio $\left({ }^{10} \mathrm{Be} /{ }^{9} \mathrm{Be}\right)$, and proton flux data respectively. There existed many attempts fitting these parameters since the release of new AMS-02 nuclei data [33, 35-41]. In particular ref. [33] combined the relevant data sets of cosmic rays measured by ACE, PAMELA and AMS02 in their Markov Chain Monte Carlo (MCMC) sampling algorithm and gave the fitted results for different propagation models. As shown in table 2, we adopt the values of propagation/injection parameters in the diffusion reacceleration (DR) model which fits both the $\mathrm{B} / \mathrm{C}$ and proton fluxes well compared with convection models and does not need additional phenomenological modification of the diffusion coefficient [33]. The values of Fisk potential are the approximate constants of the time-varying modulation form employed in the references [33, 36].

Compared to measured data, the benchmark model of propagation generally underproduces the antiproton cosmic ray at high energies. The dark matter annihilation can also produce antiprotons so as to compensate this discrepancy. The dark matter source term contributing to the cosmic ray species $i$ is given by

$$
Q_{i}^{\mathrm{DM}}(r, p)=\frac{\rho_{\mathrm{DM}}^{2}(r)\left\langle\sigma_{\mathrm{ann}} v\right\rangle}{a m_{\mathrm{DM}}^{2}} \frac{d N_{i}}{d E}, \quad i=\bar{p} \text { here }
$$


where $a=2$ (4) for self-conjugate (non-self-conjugate) dark matter. We use a generalized Navarro-Frenk-White (NFW) profile to describe dark matter spatial distribution $[42,43]$

$$
\rho_{\mathrm{DM}}(r)=\rho_{0} \frac{\left(r / r_{s}\right)^{-\gamma}}{\left(1+r / r_{s}\right)^{3-\gamma}} .
$$

The NFW profile is a traditional benchmark choice motivated by N-body simulations. The inner slope of the halo profile is chosen to be $\gamma=1$ and the radius of the galactic diffusion disk is $r_{s}=20 \mathrm{kpc}$. The coefficient $\rho_{0}$ is thus set to be $0.26 \mathrm{GeV} / \mathrm{cm}^{3}$ to give the local dark matter density $\rho_{\mathrm{DM}}\left(r_{\odot}\right)=0.3 \mathrm{GeV} / \mathrm{cm}^{3}[36]$.

As mentioned above, for each set of dark matter mass and mediator mass, we first generate the antiproton spectrum $d N_{\bar{p}} / d E$, and calculate the dark matter annihilation cross section. These dark matter model dependent variables are then passed into the public code Galprop v54 [44-48] to ensure that near Earth cosmic ray fluxes from dark matter annihilation and background are obtained in a consistent way [49]. Thus the free parameters in our analysis are the corresponding dark matter mass and mediator mass for each simplified model. The obtained cosmic ray fluxes, together with the experimental data points, are put into a composite likelihood function, defined as

$$
-2 \ln \mathcal{L}=\sum_{i} \frac{\left(f_{i}^{\text {th }}-f_{i}^{\text {exp }}\right)^{2}}{\sigma_{i}^{2}} .
$$

Here $f_{i}^{\text {th }}$ are the theoretical predictions and $f_{i}^{\text {exp }}$ are the corresponding central value of the measured data. We stipulate a $50 \%$ uncertainty of the theoretical prediction according to the estimates in refs. [36, 50-52]. This 50\% takes into account, amongst other, the uncertainty related to the fixed propagation parameters. The theoretical and experimental uncertainties are then combined in quadrature to give the $\sigma_{i}$. The sum in quadrature may lead to underestimated total error if the statistical and systematic errors are correlated, which makes our constraints become somewhat stronger than that taking into account the correlation. Note that the AMS-02 analyses of antiproton flux and antiproton-to-proton ratio were based on the same antiproton events. Although their systematic uncertainties are different, the two data sets might be correlated. In order to avoid the possible correlation, the sum in eq. (3.9) runs over only the AMS-02 antiproton flux data (57 points).

Both the explanation for the antiproton measurements and the modeling of antiproton background are the subject of debate. There exist other explanations for the difference between the data and prediction except for the dark matter annihilation. We comment on other popular antiproton explanations and background models here. The secondary antiprotons, in addition to the standard background components, can also arise from the collisions of protons accelerated by the supernova remnants (SNRs) and those in a gas cloud surrounding some local SNR $[38,53-55]$. Both the overabundant positrons and the flat $\bar{p} / p$ fraction can be explained in this local SNR scenario. To indicate the spectrum from a local supernova explosion, one needs to introduce more free parameters in an additional power law with an exponential energy cutoff $[54,55]$, i.e. $A_{i}^{\prime}, \alpha, E_{\text {cut }}$ in $A_{i}^{\prime}\left(R / R_{0}\right)^{-\alpha} \exp \left(-E / E_{\text {cut }}\right)$, as well as the parameters for the distance between the local supernova and the Sun and the interaction time between cosmic rays and the gas cloud. More precise measurements 
for cosmic ray spectrum and anisotropy are needed to validate and identify the local SNR hypothesis with different ages [55].

The background modeling of secondary antiproton is particularly established by the determination of the propagation parameters. Among the propagation parameters, the choice of convection and/or reacceleration effect governs the diffusive nature of cosmic rays in the Milky Way and helps to categorize different kinds of propagation models. The convective winds in the galaxy suggest the convective transport of cosmic rays, described by the convection velocity $\vec{V}$ in eq. (3.1). The reacceleration effect induces a momentum diffusion as shown in eq. (3.2). Recently ref. [33] discussed various propagation models with the new observation data. They employ the plain diffusion model without both reacceleration and convection, and the models with reacceleration and/or convection. The diffusion reacceleration scenario is found to be best consistent with the data of $\mathrm{B} / \mathrm{C}$ and proton. The failure of non-reacceleration models implies that the presence of convective winds in the galaxy is physically speculative $[31,56]$. We thus work in the diffusion reacceleration framework for the cosmic ray propagation as specified before. Nevertheless, we should keep in mind that the conclusion of the constraints on dark matter signal below is dependent on the background model adopted here.

\subsection{Dwarf galaxy constraint from Fermi-LAT}

For individual dwarf galaxy target, Fermi-LAT tabulated the delta-log-likelihood values as a function of the energy flux bin-by-bin [10] and newly reported an update in ref. [11]. The gamma ray energy flux from dark matter annihilation for $j$ th energy bin is given by

$$
\Phi_{j, k}^{E}\left(m_{\mathrm{DM}},\left\langle\sigma_{\mathrm{ann}} v\right\rangle, J_{k}\right)=\frac{1}{4 \pi} \frac{\left\langle\sigma_{\mathrm{ann}} v\right\rangle}{a m_{\mathrm{DM}}^{2}} J_{k} \int_{E_{j}^{\min }}^{E_{j}^{\max }} E \frac{d N_{i}}{d E} d E, \quad i=\gamma \text { here }
$$

where $J_{k}$ is the $\mathrm{J}$ factor for $k$ th dwarf. One can see that the energy flux is only dependent on three parameters, i.e. $m_{\mathrm{DM}},\left\langle\sigma_{\mathrm{ann}} v\right\rangle$ and $J_{k}$, and calculable for any dark matter annihilating process induced by the above simplified models. The likelihood for $k$ th dwarf is

$$
\mathcal{L}_{k}\left(m_{\mathrm{DM}},\left\langle\sigma_{\mathrm{ann}} v\right\rangle, J_{k}\right)=\mathcal{L}_{J}\left(J_{k} \mid \bar{J}_{k}, \sigma_{k}\right) \prod_{j} \mathcal{L}_{j, k}\left(\Phi_{j, k}^{E}\left(m_{\mathrm{DM}},\left\langle\sigma_{\mathrm{ann}} v\right\rangle, J_{k}\right)\right)
$$

where $\mathcal{L}_{j, k}$ is the tabulated likelihood provided by Fermi-LAT for each dwarf and calculated energy flux and the uncertainty of the $\mathrm{J}$ factors is taken into account by profiling over $J_{k}$ in the likelihood below

$$
\mathcal{L}_{J}\left(J_{k} \mid \bar{J}_{k}, \sigma_{k}\right)=\frac{1}{\ln (10) J_{k} \sqrt{2 \pi} \sigma_{k}} \times e^{-\left(\log _{10}\left(J_{k}\right)-\log _{10}\left(\bar{J}_{k}\right)\right)^{2} / 2 \sigma_{k}^{2}}
$$

with the measured J factor $\bar{J}_{k}$ and error $\sigma_{k}$. This likelihood form was adopted by FermiLAT collaboration in eq. (3) of ref. [10] and appeared in other literatures such as ref. [12] etc. A joint likelihood for all dwarfs is then performed as

$$
\mathcal{L}\left(m_{\mathrm{DM}},\left\langle\sigma_{\mathrm{ann}} v\right\rangle, \mathbb{J}\right)=\prod_{k} \mathcal{L}_{k}\left(m_{\mathrm{DM}},\left\langle\sigma_{\mathrm{ann}} v\right\rangle, J_{k}\right)
$$


where $\mathbb{J}$ is the set of $\mathrm{J}$ factors $J_{k}$. In our implementation we adopt the corresponding values of $\mathcal{L}_{j, k}$ and $\bar{J}_{k}, \sigma_{k}$ for 19 dwarf galaxies considered in ref. [11]. Specifically they are Bootes I, Canes Venatici I, Canes Venatici II, Carina, Coma Berenices, Draco, Fornax, Hercules, Leo I, Leo II, Leo IV, Leo V, Reticulum II, Sculptor, Segue 1, Sextans, Ursa Major I, Ursa Major II, and Ursa Minor.

As Fermi-LAT found no gamma ray excess from the dSphs, one can set upper limit on the annihilation cross section for a given $m_{\mathrm{DM}}$ by taking $\mathrm{J}$ factors as nuisance parameters in the maximum likelihood analysis. Following Fermi's approach, the delta-log-likelihood is given by

$$
-2 \Delta \ln \mathcal{L}\left(m_{\mathrm{DM}},\left\langle\sigma_{\mathrm{ann}} v\right\rangle\right)=-2 \ln \left(\frac{\mathcal{L}\left(m_{\mathrm{DM}},\left\langle\sigma_{\mathrm{ann}} v\right\rangle, \widehat{\widehat{\mathbb{J}}}\right)}{\mathcal{L}\left(m_{\mathrm{DM}},\left\langle\widehat{\sigma_{\mathrm{ann}} v}, \widehat{\mathbb{J}}\right)\right.}\right)
$$

where $\left\langle\widehat{\sigma_{\text {ann }} v}\right\rangle$ and $\widehat{\mathbb{J}}$ maximize the likelihood at any given $m_{\mathrm{DM}}$, and $\widehat{\mathbb{J}}$ maximize the likelihood for given $m_{\mathrm{DM}}$ and $\left\langle\sigma_{\mathrm{ann}} v\right\rangle$. The 95\% C.L. upper limit on annihilation cross section for a given $m_{\mathrm{DM}}$ is determined by demanding $-2 \Delta \ln \mathcal{L}\left(m_{\mathrm{DM}},\left\langle\sigma_{\mathrm{ann}} v\right\rangle\right) \leq 2.71$. We perform the likelihood analysis using Minuit [57]. If the annihilation cross section calculated by a certain set of $m_{\mathrm{DM}}$ and the mediator mass is greater than the upper limit, we claim the corresponding set of $m_{\mathrm{DM}}, m_{\mathrm{Med}}$ is excluded by Fermi-LAT dSphs.

\section{Results}

As varying the two mass parameters in simplified dark matter models and thus the likelihood function in eq. (3.9), we can fit the AMS-02 antiproton flux data and obtain the confidence regions of dark matter model parameters. We calculate $2 \sigma$ confidence region by increasing the likelihood function from its best fit value, whilst scanning the two mass parameters, until $-2 \ln \mathcal{L}$ changes by 6.18 . The dark matter contributions to the observables are then calculated using the dark matter model parameters in the $2 \sigma$ confidence region. Our likelihood function does not include the antiproton-to-proton ratio data. Rather, after we extract the dark matter model parameters, the antiproton-to-proton ratio using the fitted parameters is given as a cross check.

In figures 1, 2, 3 and 4 we show that the AMS-02 antiproton data are consistent with the dark matter framework within the uncertainties for the four models $D_{2}, D_{4}, C$ and $V$ respectively. The left plot in each figure displays the antiproton flux and the right one is the calculated antiproton-to-proton ratio. AMS-02 central values are shown by red dots and the error bars in black indicate experimental uncertainties. The green solid curves are obtained using the parameters shown in table 2 and display the predicted background originating from standard diffusion process. The dark matter contributions to the observables are then added to the background flux and give the total cosmic ray flux with dark matter contribution that fit the AMS-02 data best (blue solid lines). The dark matter contribution at the best fit point is denoted by purple curve. The combination of the background flux and the dark matter contributions calculated using the parameters in the $2 \sigma$ confidence region gives the theoretical uncertainties of the dark matter prediction (salmon colored vertical bars). 

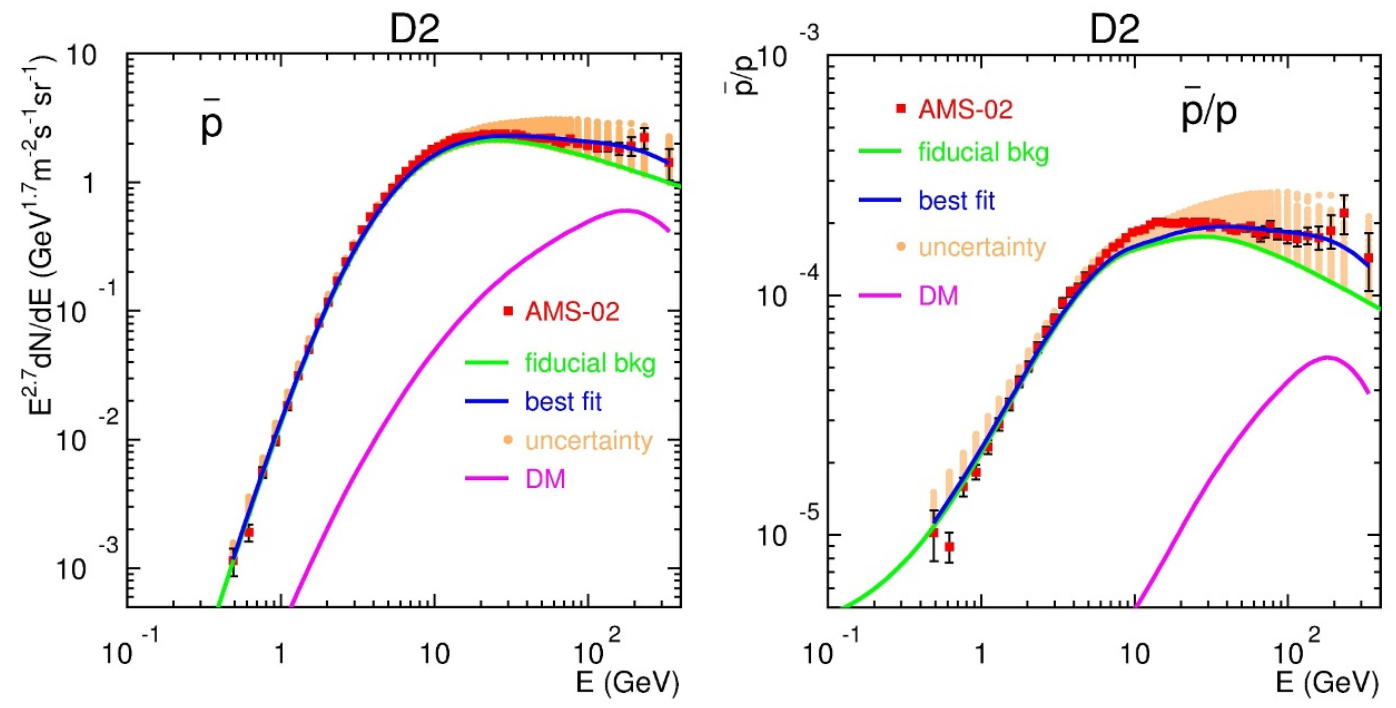

Figure 1. Antiproton flux (left) and antiproton-to-proton ratio (right) observed by AMS-02 (red dots and dark error bars) in the simplified dark matter model $D_{2}$. The blue solid line shows the prediction of the total cosmic ray flux with dark matter parameter values that best fit the AMS-02 data. The total predicted flux is the sum of the background flux (green curve) and the dark matter contribution (purple curve). Salmon dots indicate the $2 \sigma$ confidence region of the prediction.
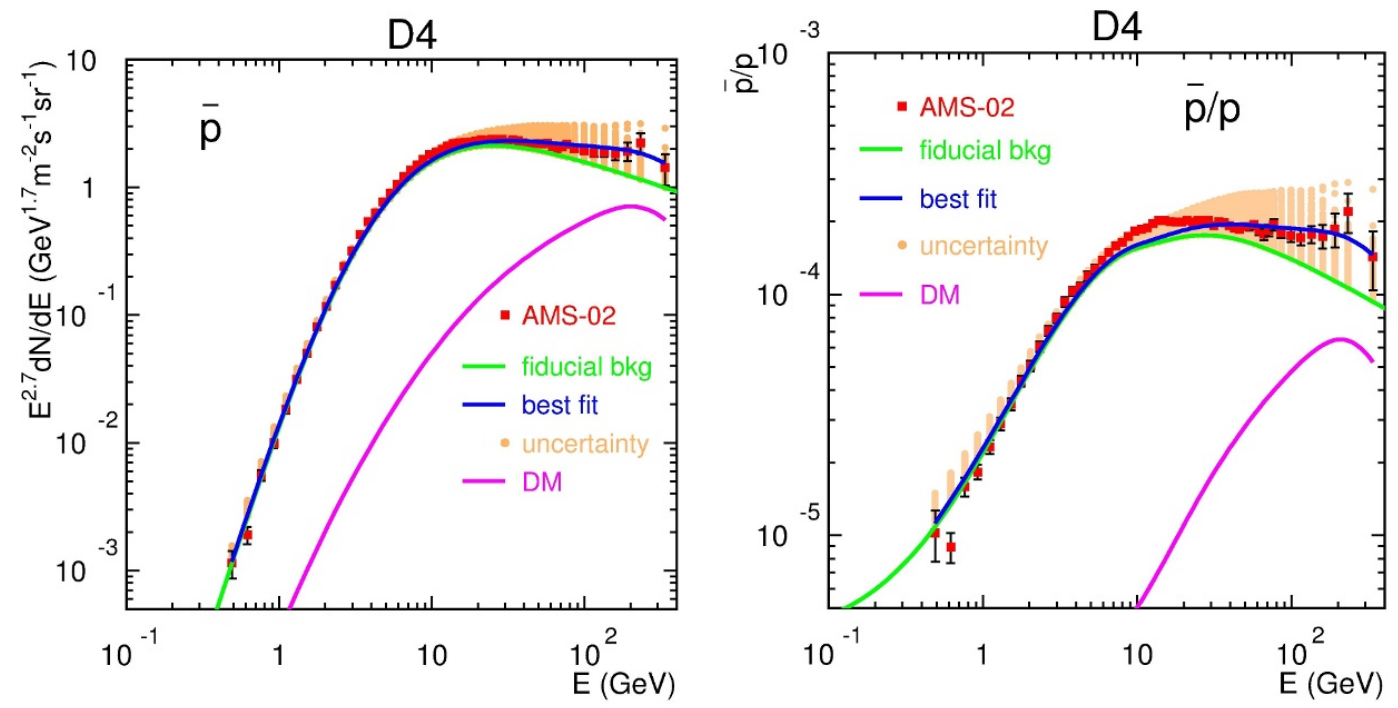

Figure 2. Antiproton flux (left) and antiproton-to-proton ratio (right) observed by AMS-02 (red dots and dark error bars) in the simplified dark matter model $D_{4}$. 

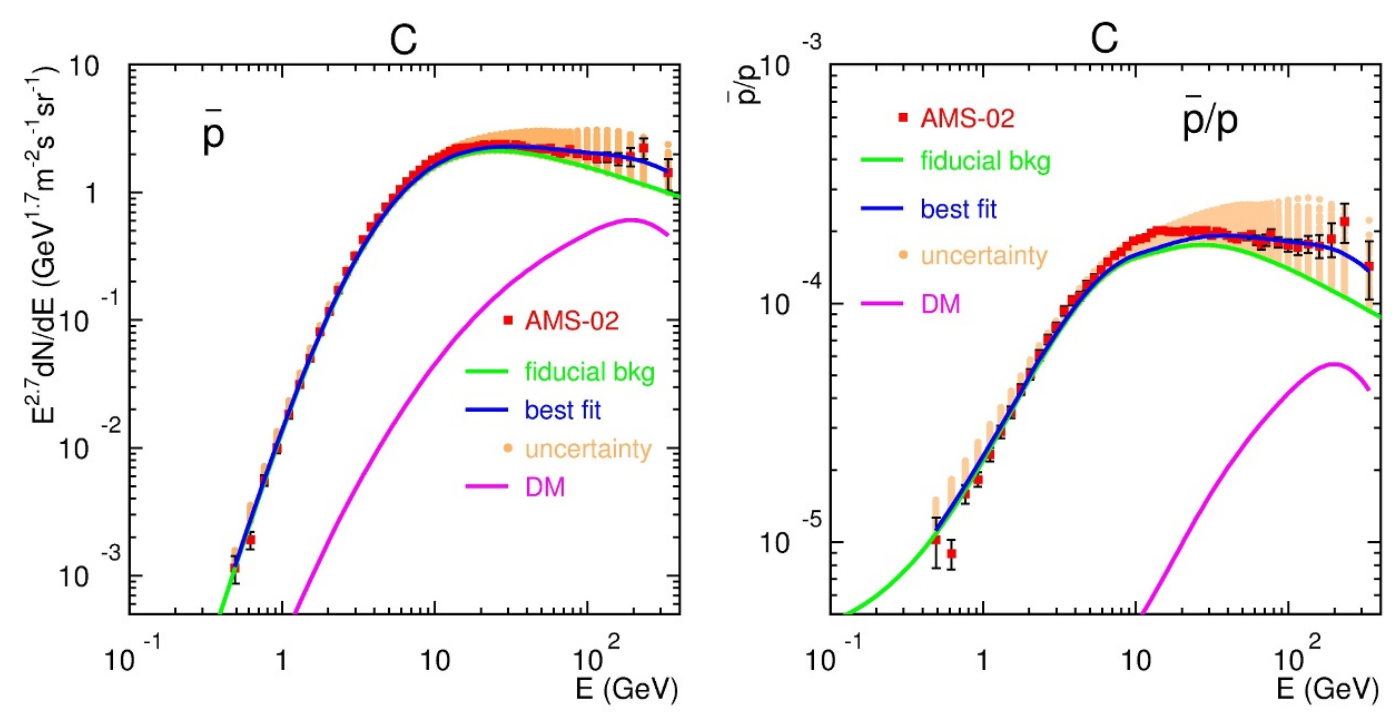

Figure 3. Antiproton flux (left) and antiproton-to-proton ratio (right) observed by AMS-02 (red dots and dark error bars) in the simplified dark matter model $C$.
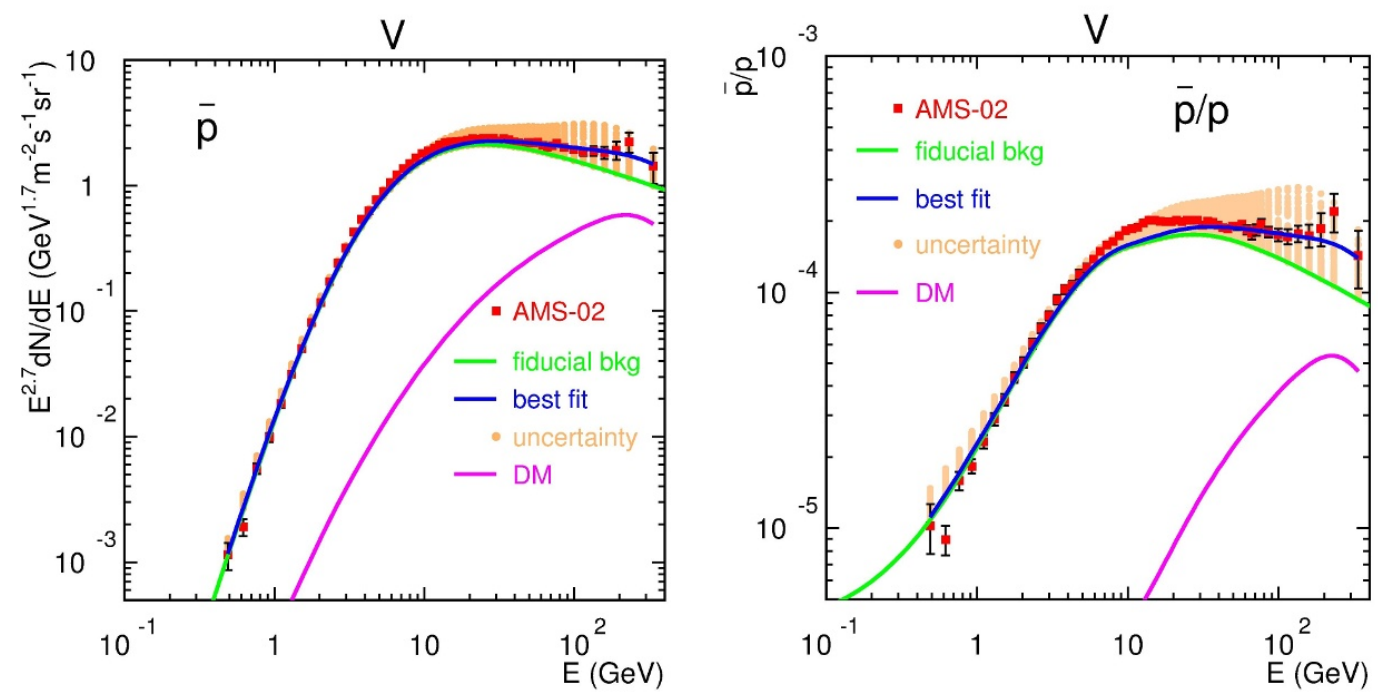

Figure 4. Antiproton flux (left) and antiproton-to-proton ratio (right) observed by AMS-02 (red dots and dark error bars) in the simplified dark matter model $V$.

Although the most stringent constraints on the simplified models we consider in principle come from indirect detection of dark matter, LHC performed dark matter search using events with large missing transverse momentum plus energetic jet for pseudo-scalar mediator model $\left(D_{4}\right)$ at $13 \mathrm{TeV}$ collisions [58]. Their exclusion limit can be directly presented in the plane of dark matter mass vs. mediator mass. As models $D_{2}$ and $D_{4}$ are closely related operators, they should have similar collider constraints and thus we can adopt the LHC constraint on model $D_{4}$ for $D_{2}$. 
In the left frames of figures 5 and 6 we show the regions of the mass parameter space preferred by the AMS-02 antiproton data and the constraints from Fermi-LAT dSphs and LHC for models $D_{2}$ and $D_{4}$, respectively. Solid squares denote the estimated $2 \sigma$ confidence region. We find that the AMS-02 antiproton data favor $30 \mathrm{GeV} \lesssim m_{\chi} \lesssim 5 \mathrm{TeV}$ region at $2 \sigma$ confidence level. The excluded regions by Fermi-LAT dSphs are denoted in red circles. The LHC excludes a small part of the $2 \sigma$ confidence region with $m_{\chi} \lesssim 170 \mathrm{GeV}$ and $200 \mathrm{GeV} \lesssim m_{S_{2}}, m_{S_{4}} \lesssim 420 \mathrm{GeV}$. For scalar and vector dark matter models, the left frames of figures 7 and 8 show that the AMS-02 antiproton data favor dark matter masses in the region of $50 \mathrm{GeV}-5 \mathrm{TeV}$.

The right frames of figures 5 and 6 show that the AMS-02 data require an effective dark matter annihilation cross section in the region of $4 \times 10^{-27}-4 \times 10^{-24} \mathrm{~cm}^{3} / \mathrm{s}$ for both $D_{2}$ and $D_{4}$ models at about $2 \sigma$ C.L. The LHC excludes a part of the low dark matter mass region, denoted by green stars. The relatively small cross section region in red circles can evade the limit from Fermi-LAT dSphs, for instance $\left\langle\sigma_{\text {ann }} v\right\rangle \lesssim 2 \times 10^{-26} \mathrm{~cm}^{3} / \mathrm{s}$ allowed for $m_{\chi} \simeq 100 \mathrm{GeV}$ and $\left\langle\sigma_{\mathrm{ann}} v\right\rangle \lesssim 2 \times 10^{-25} \mathrm{~cm}^{3} / \mathrm{s}$ for $m_{\chi} \simeq 1 \mathrm{TeV}$. The annihilation cross section region $7 \times 10^{-27}-4 \times 10^{-24} \mathrm{~cm}^{3} / \mathrm{s}$ is favored for scalar and vector dark matter as displayed in the right frames of figures 7 and 8 .

As seen from the right frames of figures 5, 6, 7 and 8, the AMS-02 data favor thermal annihilation cross section. The left frames of them generally show a double-leg structure in the plane of $M_{\mathrm{DM}}$ vs. $M_{\mathrm{Med}}$. They agree with the thermal relic density calculation [20]. The double-leg behavior can be understood by different kinematic features of various dark matter annihilations, required by the expected relic density. The annihilation cross sections into SM quarks are proportional to $m_{q}^{2}$, while the annihilation into mediator pairs simply depends on the ratio $M_{\mathrm{Med}} / M_{\mathrm{DM}}$. When $M_{\mathrm{DM}}>M_{\mathrm{Med}}$, the annihilation cross section is governed by $M_{\mathrm{Med}} / M_{\mathrm{DM}}$, which explains the flat distributions for $M_{\mathrm{DM}} \lesssim 2 \mathrm{TeV}$. When the dark matter mass becomes smaller than the mediator mass, in particular once the $t \bar{t}$ channel is kinematically allowed, the annihilation cross section into SM quarks becomes efficient and then exhibits the other legs and the kinks in the plots.

Next, we comment on the background model uncertainties which mainly come from the collision cross sections of primary cosmic rays with the interstellar medium and the diffusion coefficients. The diffusion coefficients of cosmic rays may vary in the Milky Way due to different magnetic field distributions in the disk and halo, and it is not realistic to calculate the hadronic processes from cosmic ray interaction with interstellar gas from QCD. Therefore, in the estimate of these uncertainties, simplified frameworks and empirical parametrization are usually assumed. Ref. [39] recently re-evaluated the uncertainty of the prediction originates from the cosmic ray propagation process and the hadronic interaction models. They find that the propagation parameters have been well controlled by AMS02 data, but the results for different hadronic models do not converge. The collider most favored hadronic model does not fit $\bar{p} / p$ data at high energies. Due to the interplay between these two uncertainty sources, we leave the full variation of parameters to a future work. Alternatively we include an effective factor $(50 \%)$ for the systematic uncertainty in our likelihood analysis to represent the above uncertainties related to the fixed parameters in table 2 . 

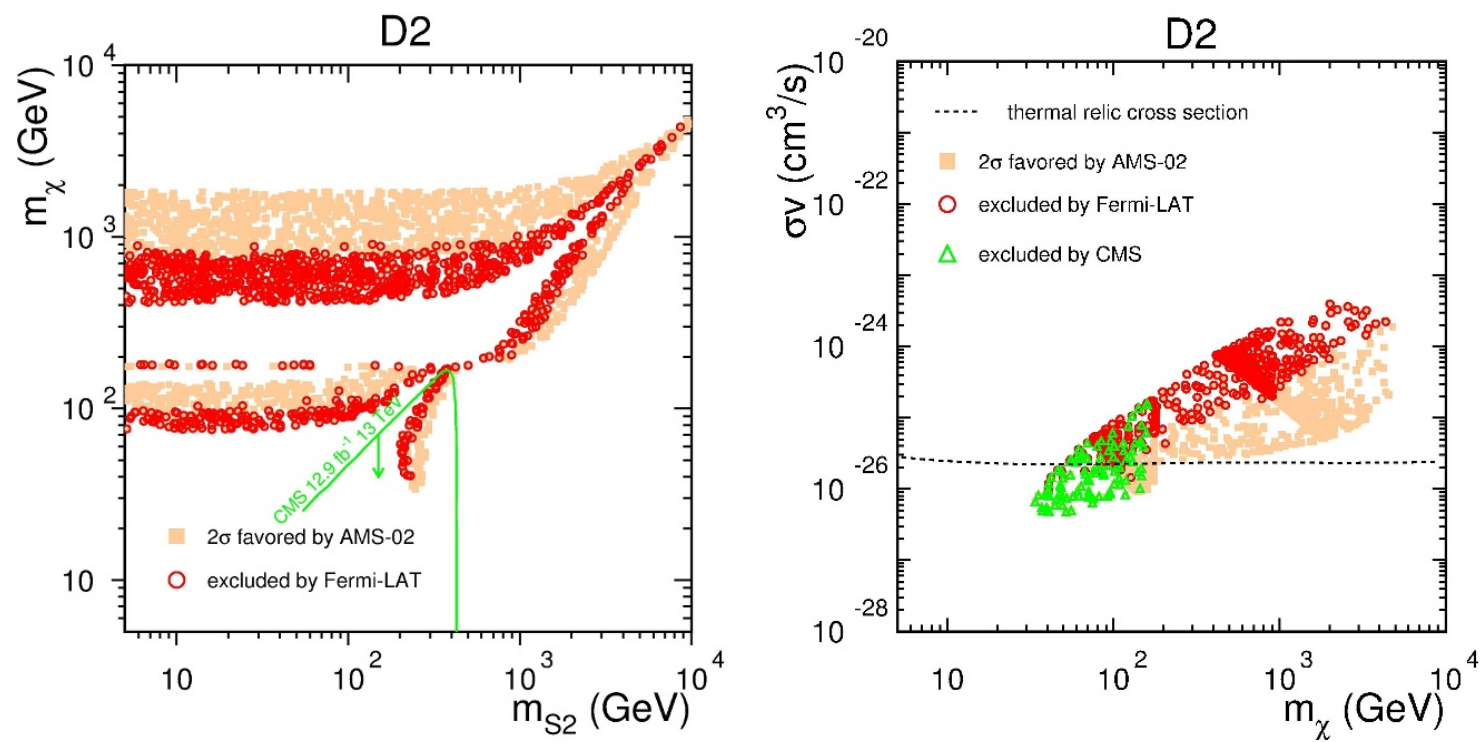

Figure 5. Left: the AMS-02 favored region of masses $\left(m_{\chi}\right.$ vs. $\left.m_{S_{2}}\right)$ in the simplified dark matter model $D_{2}$ we consider. The solid squares estimate $2 \sigma$ confidence region. The green curve is the LHC exclusion limit [58]. Right: the AMS-02 favored region of cross sections $\left(\sigma v\right.$ vs. $\left.m_{\chi}\right)$. The green points are excluded by LHC search. The red circles are excluded by Fermi-LAT dSphs. The black dashed curve corresponds to the thermal cross section [59].
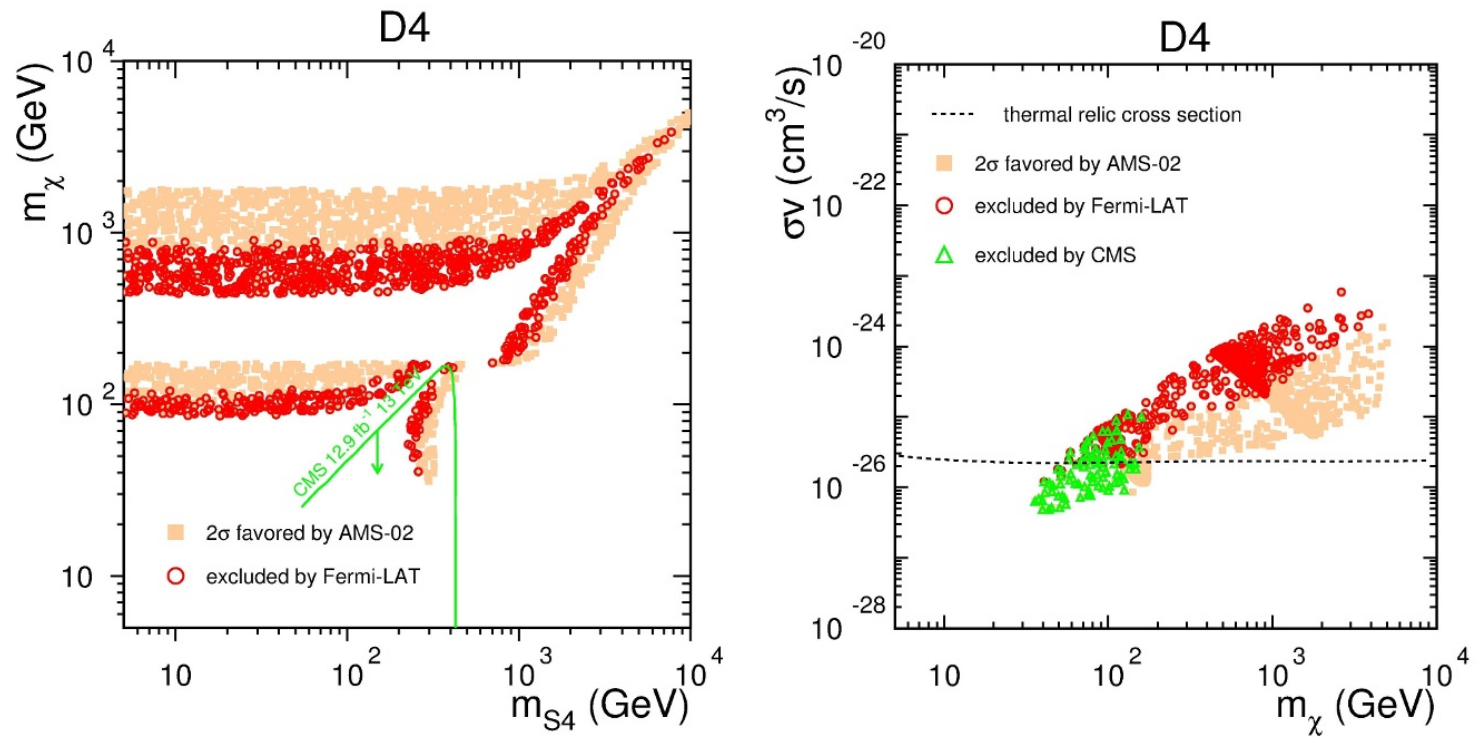

Figure 6. Left: the AMS-02 favored region of masses $\left(m_{\chi}\right.$ vs. $\left.m_{S_{4}}\right)$ in the simplified dark matter model $D_{4}$. Right: the AMS-02 favored region of cross sections ( $\sigma v$ vs. $\left.m_{\chi}\right)$. 

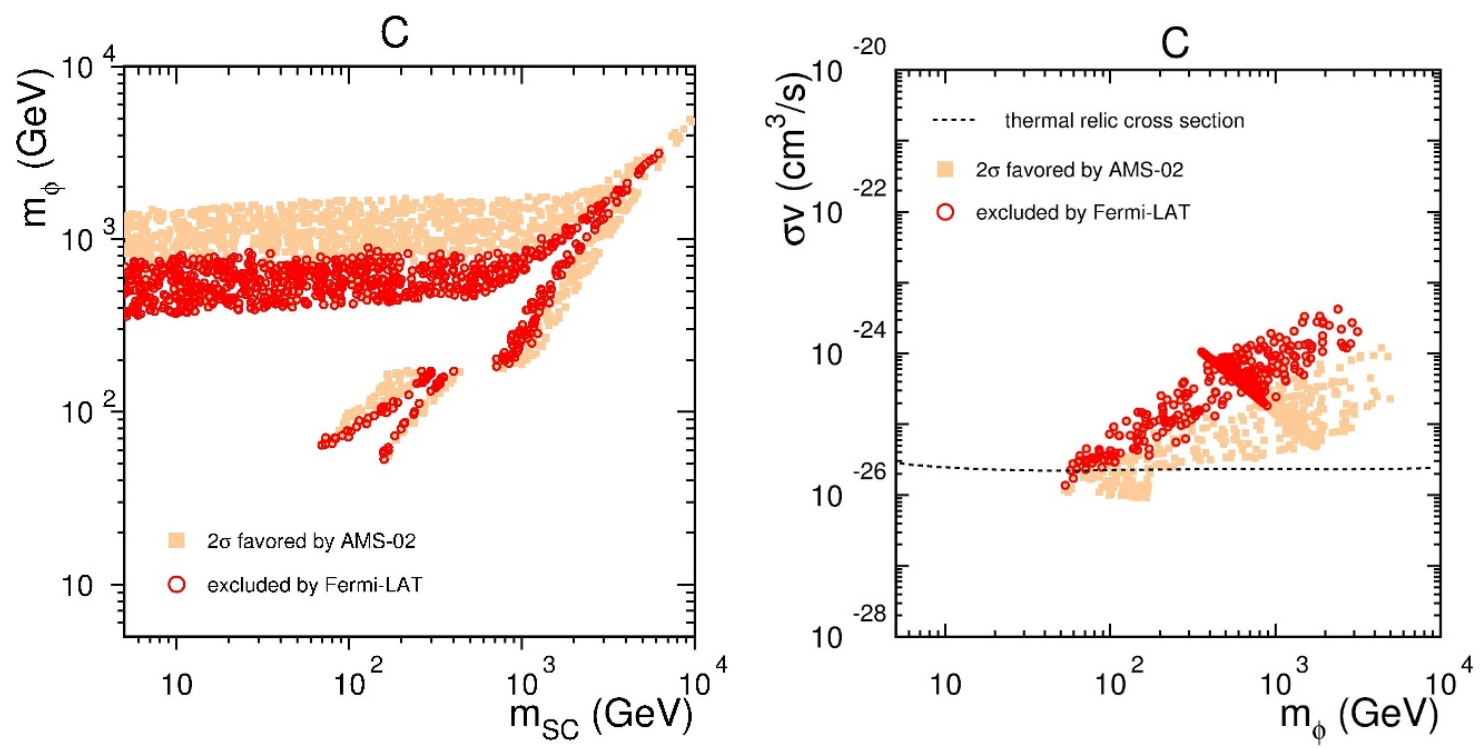

Figure 7. Left: the AMS-02 favored region of masses $\left(m_{\phi}\right.$ vs. $\left.m_{S_{C}}\right)$ in the simplified dark matter model $C$. Right: the AMS-02 favored region of cross sections ( $\sigma v$ vs. $\left.m_{\phi}\right)$.
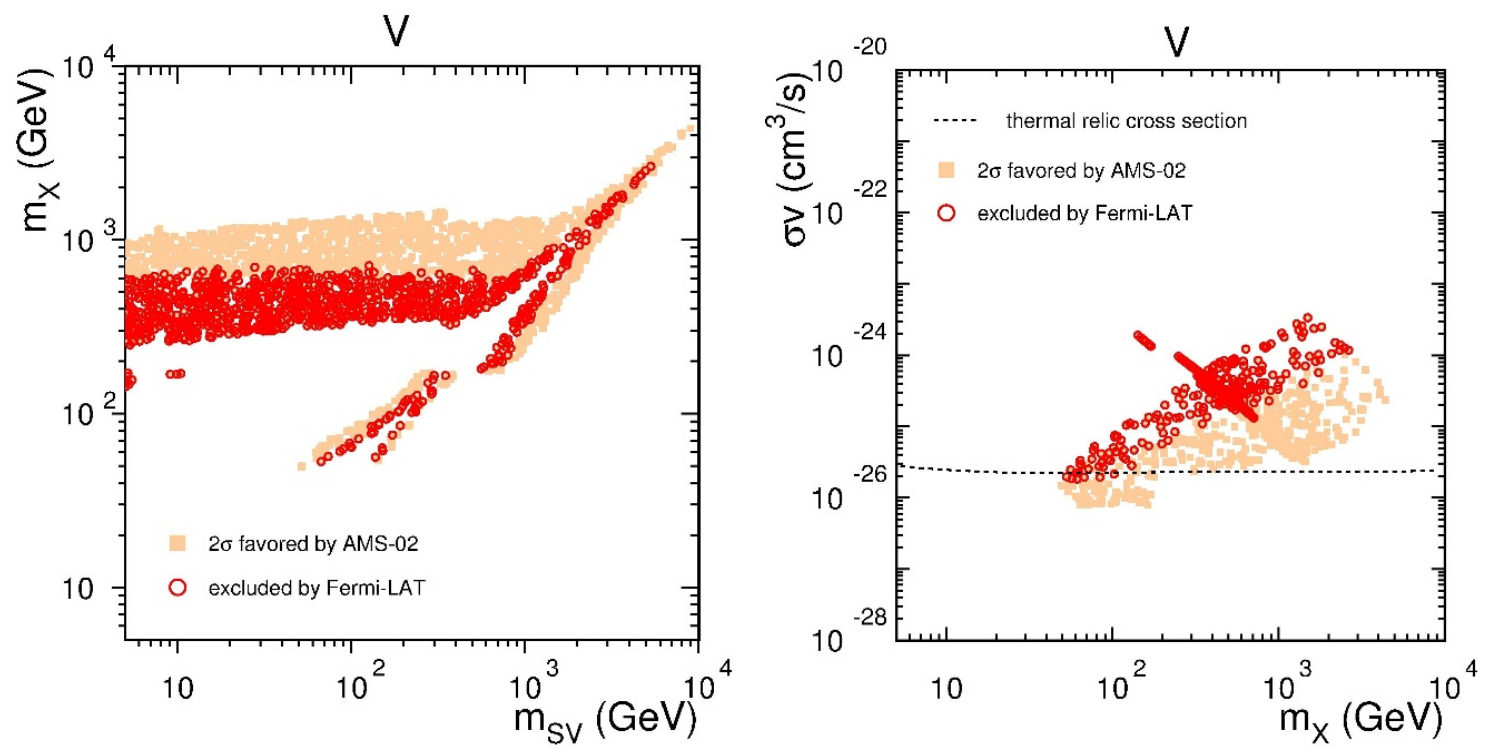

Figure 8. Left: the AMS-02 favored region of masses $\left(m_{X}\right.$ vs. $\left.m_{S_{V}}\right)$ in the simplified dark matter model $V$. Right: the AMS-02 favored region of cross sections ( $\sigma v$ vs. $m_{X}$ ). 


\section{Conclusions}

In this work we investigate the simplified dark matter models favored by the recent AMS02 antiproton data and consider the constraint from no gamma ray excess in Milky Way dSphs. The propagation and injection parameters of cosmic rays are determined by fitting the latest AMS-02 data of nuclei fluxes and the secondary antiproton flux is obtained as the fiducial background. In addition to the standard astrophysical cosmic ray, we include a fermion, scalar or vector dark matter component from four simplified models with leptophobic spin-0 mediators that couple only to SM quarks and dark matter particles via scalar and/or pseudo-scalar bilinear. The WIMP-nucleon scattering cross sections and the events with large missing energy plus energetic jet at collider search are both suppressed for the simplified models we consider.

We have shown that the dark matter contribution to the background flux gives a better fit to the data. The observation of antiproton prefers dark matter masses in the region of $30(50) \mathrm{GeV}-5 \mathrm{TeV}$ for simplified fermion (scalar and vector) dark matter models at about $2 \sigma$ confidence level. The AMS-02 data also require fermion (scalar and vector) dark matter annihilation cross section as $4 \times 10^{-27}\left(7 \times 10^{-27}\right)-4 \times 10^{-24} \mathrm{~cm}^{3} / \mathrm{s}$. The LHC excludes a part of the favored region with $m_{\chi} \lesssim 170 \mathrm{GeV}$ for fermion dark matter models. The relatively small cross section region can evade the limit from Fermi-LAT dSphs, for instance $\left\langle\sigma_{\text {ann }} v\right\rangle \lesssim 2 \times 10^{-26} \mathrm{~cm}^{3} / \mathrm{s}$ for about $100 \mathrm{GeV}$ dark matter mass.

Finally, due to the presence of different explanations for the antiproton measurements, we conclude that the positive indications for a dark matter signal depend strongly on the assumed background model, and is specific to the model adopted in this work.

\section{Acknowledgments}

We would like to thank Yi Cai for helping with Minuit. The National Computational Infrastructure (NCI), the Southern Hemisphere's fastest supercomputer, is also gratefully acknowledged.

\section{A Expressions of mediator decay widths and dark matter annihilation cross sections}

\section{A.1 fermion dark matter model $D_{2}$}

The mediator decay widths for the $S_{2}$ mediator case $[16,17]$ :

$$
\begin{aligned}
\Gamma_{S_{2} \rightarrow \bar{\chi} \chi} & =\frac{\left(g_{\chi}^{\mathrm{D} 2}\right)^{2} m_{S_{2}}}{8 \pi}\left(1-\frac{4 m_{\chi}^{2}}{m_{S_{2}}^{2}}\right)^{1 / 2}, \\
\Gamma_{S_{2} \rightarrow \bar{q} q} & =N_{c} \frac{\left(g_{q}^{\mathrm{D} 2}\right)^{2} m_{S_{2}}}{8 \pi} \frac{m_{q}^{2}}{v_{0}^{2}}\left(1-\frac{4 m_{q}^{2}}{m_{S_{2}}^{2}}\right)^{3 / 2} q=u, d, s, c, b, t, \\
\Gamma_{S_{2} \rightarrow g g} & =\frac{\left(g_{q}^{\mathrm{D} 2}\right)^{2} \alpha_{s}^{2}\left(m_{S_{2}}\right) m_{S_{2}}^{3}}{32 \pi^{3} v_{0}^{2}}\left|\frac{4 m_{t}^{2}}{m_{S_{2}}^{2}}\left[1+\left(1-\frac{4 m_{t}^{2}}{m_{S_{2}}^{2}}\right) \arctan ^{2}\left(\left(\frac{4 m_{t}^{2}}{m_{S_{2}}^{2}}-1\right)^{-1 / 2}\right)\right]\right|^{2}, \\
\Gamma_{S_{2}} & =\Gamma_{S_{2} \rightarrow \bar{\chi} \chi}+\Gamma_{S_{2} \rightarrow \bar{q} q}+\Gamma_{S_{2} \rightarrow g g}
\end{aligned}
$$


The dark matter annihilation cross sections for the $S_{2}$ mediator case:

$$
\begin{aligned}
\sigma_{\mathrm{ann}} v\left(\bar{\chi} \chi \rightarrow S_{2} \rightarrow \bar{q} q\right) & =\frac{\left(g_{\chi}^{\mathrm{D} 2}\right)^{2}\left(g_{q}^{\mathrm{D} 2}\right)^{2} N_{c}}{\left(4 m_{\chi}^{2}-m_{S_{2}}^{2}\right)^{2}+m_{S_{2}}^{2} \Gamma_{S_{2}}^{2}} \frac{m_{\chi}^{2}}{2 \pi} \frac{m_{q}^{2}}{v_{0}^{2}}\left(1-\frac{m_{q}^{2}}{m_{\chi}^{2}}\right)^{3 / 2}, \\
\sigma_{\mathrm{ann}} v\left(\bar{\chi} \chi \rightarrow S_{2} S_{2}\right) & =\left(g_{\chi}^{\mathrm{D} 2}\right)^{4} \frac{m_{\chi}^{2}\left(m_{\chi}^{4}-2 m_{\chi}^{2} m_{S_{2}}^{2}+m_{S_{2}}^{4}\right)}{24 \pi\left(2 m_{\chi}^{2}-m_{S_{2}}^{2}\right)^{4}}\left(1-\frac{m_{S_{2}}^{2}}{m_{\chi}^{2}}\right)^{1 / 2} v^{2},
\end{aligned}
$$

where $v \simeq 10^{-3}$.

\section{A.2 fermion dark matter model $D_{4}$}

The mediator decay widths for the $S_{4}$ mediator case [17]:

$$
\begin{aligned}
\Gamma_{S_{4} \rightarrow \bar{\chi} \chi} & =\frac{\left(g_{\chi}^{\mathrm{D} 4}\right)^{2} m_{S_{4}}}{8 \pi}\left(1-\frac{4 m_{\chi}^{2}}{m_{S_{4}}^{2}}\right)^{1 / 2}, \\
\Gamma_{S_{4} \rightarrow \bar{q} q} & =N_{c} \frac{\left(g_{q}^{\mathrm{D} 4}\right)^{2} m_{S_{4}}}{8 \pi} \frac{m_{q}^{2}}{v_{0}^{2}}\left(1-\frac{4 m_{q}^{2}}{m_{S_{4}}^{2}}\right)^{1 / 2} q=u, d, s, c, b, t, \\
\Gamma_{S_{4} \rightarrow g g} & =\frac{\left(g_{q}^{\mathrm{D} 4}\right)^{2} \alpha_{s}^{2}\left(m_{S_{4}}\right) m_{S_{4}}^{3}}{32 \pi^{3} v_{0}^{2}}\left|\frac{4 m_{t}^{2}}{m_{S_{4}}^{2}} \arctan ^{2}\left(\left(\frac{4 m_{t}^{2}}{m_{S_{4}}^{2}}-1\right)^{-1 / 2}\right)\right|^{2}, \\
\Gamma_{S_{4}} & =\Gamma_{S_{4} \rightarrow \bar{\chi} \chi}+\Gamma_{S_{4} \rightarrow \bar{q} q}+\Gamma_{S_{4} \rightarrow g g}
\end{aligned}
$$

The dark matter annihilation cross sections for the $S_{4}$ mediator case [14]:

$$
\begin{aligned}
\sigma_{\mathrm{ann}} v\left(\bar{\chi} \chi \rightarrow S_{4} \rightarrow \bar{q} q\right) & =\frac{\left(g_{\chi}^{\mathrm{D} 4}\right)^{2}\left(g_{q}^{\mathrm{D} 4}\right)^{2} N_{c}}{\left(4 m_{\chi}^{2}-m_{S_{4}}^{2}\right)^{2}+m_{S_{4}}^{2} \Gamma_{S_{4}}^{2}} \frac{m_{\chi}^{2}}{2 \pi} \frac{m_{q}^{2}}{v_{0}^{2}}\left(1-\frac{m_{q}^{2}}{m_{\chi}^{2}}\right)^{1 / 2}, \\
\sigma_{\mathrm{ann}} v\left(\bar{\chi} \chi \rightarrow S_{4} S_{4}\right) & =\left(g_{\chi}^{\mathrm{D} 4}\right)^{4} \frac{m_{\chi}^{2}\left(m_{\chi}^{4}-2 m_{\chi}^{2} m_{S_{4}}^{2}+m_{S_{4}}^{4}\right)}{24 \pi\left(2 m_{\chi}^{2}-m_{S_{4}}^{2}\right)^{4}}\left(1-\frac{m_{S_{4}}^{2}}{m_{\chi}^{2}}\right)^{1 / 2} v^{2},
\end{aligned}
$$

where $v \simeq 10^{-3}$.

\section{A.3 scalar dark matter model $C$}

The $S_{C}$ mediator decay widths for the scalar dark matter case [60]:

$$
\begin{aligned}
\Gamma_{S_{C} \rightarrow \phi^{\dagger} \phi} & =\frac{\left(g_{\phi}^{\mathrm{C}}\right)^{2} m_{\phi}^{2}}{16 \pi m_{S_{C}}}\left(1-\frac{4 m_{\phi}^{2}}{m_{S_{C}}^{2}}\right)^{1 / 2}, \\
\Gamma_{S_{C} \rightarrow \bar{q} q} & =N_{c} \frac{\left(g_{q}^{\mathrm{C}}\right)^{2} m_{S_{C}}}{8 \pi} \frac{m_{q}^{2}}{v_{0}^{2}}\left(1-\frac{4 m_{q}^{2}}{m_{S_{C}}^{2}}\right)^{1 / 2} q=u, d, s, c, b, t, \\
\Gamma_{S_{C} \rightarrow g g} & =\frac{\left(g_{q}^{\mathrm{C}}\right)^{2} \alpha_{s}^{2}\left(m_{S_{C}}\right) m_{S_{C}}^{3}}{32 \pi^{3} v_{0}^{2}}\left|\frac{4 m_{t}^{2}}{m_{S_{C}}^{2}} \arctan ^{2}\left(\left(\frac{4 m_{t}^{2}}{m_{S_{C}}^{2}}-1\right)^{-1 / 2}\right)\right|^{2}, \\
\Gamma_{S_{C}} & =\Gamma_{S_{C} \rightarrow \phi^{\dagger} \phi}+\Gamma_{S_{C} \rightarrow \bar{q} q}+\Gamma_{S_{C} \rightarrow g g}
\end{aligned}
$$


The dark matter annihilation cross sections for the $S_{C}$ mediator case [60]:

$$
\begin{aligned}
\sigma_{\mathrm{ann}} v\left(\phi^{\dagger} \phi \rightarrow S_{C} \rightarrow \bar{q} q\right) & =\frac{\left(g_{\phi}^{\mathrm{C}}\right)^{2}\left(g_{q}^{\mathrm{C}}\right)^{2} N_{c}}{\left(4 m_{\phi}^{2}-m_{S_{C}}^{2}\right)^{2}+m_{S_{C}}^{2} \Gamma_{S_{C}}^{2}} \frac{m_{\phi}^{2} m_{q}^{2}}{4 \pi v_{0}^{2}}\left(1-\frac{m_{q}^{2}}{m_{\phi}^{2}}\right)^{1 / 2}, \\
\sigma_{\mathrm{ann}} v\left(\phi^{\dagger} \phi \rightarrow S_{C} S_{C}\right) & =\frac{\left(g_{\phi}^{\mathrm{C}}\right)^{4} m_{\phi}\left(m_{\phi}^{2}-m_{S_{C}}^{2}\right)^{1 / 2}}{16 \pi\left(2 m_{\phi}^{2}-m_{S_{C}}^{2}\right)^{2}} .
\end{aligned}
$$

\section{A.4 vector dark matter model $V$}

The $S_{V}$ mediator decay widths for the vector dark matter case [61]:

$$
\begin{gathered}
\Gamma_{S_{V} \rightarrow X^{\dagger} X}=\frac{\left(g_{X}^{\mathrm{V}}\right)^{2} m_{S_{V}}^{3}}{64 \pi m_{X}^{2}}\left(1-\frac{4 m_{X}^{2}}{m_{S_{V}}^{2}}+\frac{12 m_{X}^{4}}{m_{S_{V}}^{4}}\right)\left(1-\frac{4 m_{X}^{2}}{m_{S_{V}}^{2}}\right)^{1 / 2}, \\
\Gamma_{S_{V} \rightarrow \bar{q} q}=N_{c} \frac{\left(g_{q}^{\mathrm{V}}\right)^{2} m_{S_{V}}}{8 \pi} \frac{m_{q}^{2}}{v_{0}^{2}}\left(1-\frac{4 m_{q}^{2}}{m_{S_{V}}^{2}}\right)^{1 / 2} q=u, d, s, c, b, t, \\
\Gamma_{S_{V} \rightarrow g g}=\frac{\left(g_{q}^{\mathrm{V}}\right)^{2} \alpha_{s}^{2}\left(m_{S_{V}}\right) m_{S_{V}}^{3}}{32 \pi^{3} v_{0}^{2}}\left|\frac{4 m_{t}^{2}}{m_{S_{V}}^{2}} \arctan ^{2}\left(\left(\frac{4 m_{t}^{2}}{m_{S_{4}}^{2}}-1\right)^{-1 / 2}\right)\right|^{2}, \\
\Gamma_{S_{V}}=\Gamma_{S_{V} \rightarrow X^{\dagger} X}+\Gamma_{S_{V} \rightarrow \bar{q} q}+\Gamma_{S_{V} \rightarrow g g}
\end{gathered}
$$

The dark matter annihilation cross sections for the $S_{V}$ mediator case [61, 62]:

$$
\begin{aligned}
\sigma_{\mathrm{ann}} v\left(X^{\dagger} X \rightarrow S_{V} \rightarrow \bar{q} q\right) & =\frac{\left(g_{X}^{\mathrm{V}}\right)^{2}\left(g_{q}^{\mathrm{V}}\right)^{2} N_{c}}{\left(4 m_{X}^{2}-m_{S_{V}}^{2}\right)^{2}+m_{S_{V}}^{2} \Gamma_{S_{V}}^{2}} \frac{m_{X}^{2} m_{q}^{2}}{12 \pi v_{0}^{2}}\left(1-\frac{m_{q}^{2}}{m_{X}^{2}}\right)^{1 / 2} \\
\sigma_{\mathrm{ann}} v\left(X^{\dagger} X \rightarrow S_{V} S_{V}\right) & =\frac{\left(g_{X}^{\mathrm{V}}\right)^{4}}{144 \pi m_{X}^{2}} \frac{6 m_{X}^{4}-4 m_{X}^{2} m_{S_{V}}^{2}+m_{S_{V}}^{4}}{\left(2 m_{X}^{2}-m_{S_{V}}^{2}\right)^{2}}\left(1-\frac{m_{S_{V}}^{2}}{m_{X}^{2}}\right)^{1 / 2}
\end{aligned}
$$

Open Access. This article is distributed under the terms of the Creative Commons Attribution License (CC-BY 4.0), which permits any use, distribution and reproduction in any medium, provided the original author(s) and source are credited.

\section{References}

[1] V.L. Ginzburg and S.I. Syrovatskii, The Origin of Cosmic Rays, Macmillan, New York, U.S.A. (1964).

[2] R. Blandford and D. Eichler, Particle Acceleration at Astrophysical Shocks: A Theory of Cosmic Ray Origin, Phys. Rept. 154 (1987) 1 [InSPIRE].

[3] L. Stawarz, V. Petrosian and R.D. Blandford, On the Energy Spectra of GeV/TeV Cosmic Ray Leptons, Astrophys. J. 710 (2010) 236 [arXiv:0908.1094] [INSPIRE].

[4] F. Aharonian, A. Bykov, E. Parizot, V. Ptuskin and A. Watson, Cosmic rays in galactic and extragalactic magnetic fields, Space Sci. Rev. 166 (2012) 97 [arXiv:1105.0131] [INSPIRE].

[5] AMS collaboration, M. Aguilar et al., Precision Measurement of the Proton Flux in Primary Cosmic Rays from Rigidity 1 GV to 1.8 TV with the Alpha Magnetic Spectrometer on the International Space Station, Phys. Rev. Lett. 114 (2015) 171103 [INSPIRE]. 
[6] AMS collaboration, M. Aguilar et al., Precision Measurement of the Boron to Carbon Flux Ratio in Cosmic Rays from 1.9 GV to 2.6 TV with the Alpha Magnetic Spectrometer on the International Space Station, Phys. Rev. Lett. 117 (2016) 231102 [InSPIRE].

[7] N.E. Yanasak et al., Measurement of the Secondary Radionuclides ${ }^{10} \mathrm{Be},{ }^{26} \mathrm{Al},{ }^{36} \mathrm{Cl},{ }^{54} \mathrm{Mn}$, and ${ }^{14} \mathrm{C}$ and Implications for the Galactic Cosmic-Ray Age, Astrophys. J. 563 (2001) 768.

[8] O. Adriani et al., Time dependence of the proton flux measured by PAMELA during the July 2006 - December 2009 solar minimum, Astrophys. J. 765 (2013) 91 [arXiv:1301.4108] [INSPIRE].

[9] AMS collaboration, M. Aguilar et al., Antiproton Flux, Antiproton-to-Proton Flux Ratio and Properties of Elementary Particle Fluxes in Primary Cosmic Rays Measured with the Alpha Magnetic Spectrometer on the International Space Station, Phys. Rev. Lett. 117 (2016) 091103 [INSPIRE].

[10] Fermi-LAT collaboration, M. Ackermann et al., Searching for Dark Matter Annihilation from Milky Way Dwarf Spheroidal Galaxies with Six Years of Fermi Large Area Telescope Data, Phys. Rev. Lett. 115 (2015) 231301 [arXiv: 1503.02641] [INSPIRE].

[11] DES and Fermi-LAT collaborations, A. Albert et al., Searching for Dark Matter Annihilation in Recently Discovered Milky Way Satellites with Fermi-LAT, Astrophys. J. 834 (2017) 110 [arXiv: 1611.03184] [INSPIRE].

[12] G. Elor, N.L. Rodd, T.R. Slatyer and W. Xue, Model-Independent Indirect Detection Constraints on Hidden Sector Dark Matter, JCAP 06 (2016) 024 [arXiv:1511.08787] [INSPIRE].

[13] O. Buchmueller, M.J. Dolan and C. McCabe, Beyond Effective Field Theory for Dark Matter Searches at the LHC, JHEP 01 (2014) 025 [arXiv: 1308.6799] [INSPIRE].

[14] C. Arina, E. Del Nobile and P. Panci, Dark Matter with Pseudoscalar-Mediated Interactions Explains the DAMA Signal and the Galactic Center Excess, Phys. Rev. Lett. 114 (2015) 011301 [arXiv: 1406 .5542] [INSPIRE].

[15] A. Alves, A. Berlin, S. Profumo and F.S. Queiroz, Dark Matter Complementarity and the $Z^{\prime}$ Portal, Phys. Rev. D 92 (2015) 083004 [arXiv:1501.03490] [InSPIRE].

[16] J. Abdallah et al., Simplified Models for Dark Matter Searches at the LHC, Phys. Dark Univ. 9-10 (2015) 8 [arXiv:1506.03116] [INSPIRE].

[17] G. Busoni et al., Recommendations on presenting LHC searches for missing transverse energy signals using simplified s-channel models of dark matter, arXiv:1603.04156 [INSPIRE].

[18] C. Arina et al., A comprehensive approach to dark matter studies: exploration of simplified top-philic models, JHEP 11 (2016) 111 [arXiv:1605.09242] [INSPIRE].

[19] A. Ismail, W.-Y. Keung, K.-H. Tsao and J. Unwin, Axial vector $Z^{\prime}$ and anomaly cancellation, Nucl. Phys. B 918 (2017) 220 [arXiv:1609.02188] [INSPIRE].

[20] C. Balázs et al., Sensitivity of the Cherenkov Telescope Array to the detection of a dark matter signal in comparison to direct detection and collider experiments, Phys. Rev. D 96 (2017) 083002 [arXiv: 1706.01505] [inSPIRE].

[21] A.J. Buras, P. Gambino, M. Gorbahn, S. Jager and L. Silvestrini, Universal unitarity triangle and physics beyond the standard model, Phys. Lett. B 500 (2001) 161 [hep-ph/0007085] [INSPIRE]. 
[22] J. Goodman, M. Ibe, A. Rajaraman, W. Shepherd, T.M.P. Tait and H.-B. Yu, Constraints on Dark Matter from Colliders, Phys. Rev. D 82 (2010) 116010 [arXiv: 1008.1783] [InSPIRE].

[23] CMS collaboration, Search for narrow resonances in dijet final states at $\sqrt{s}=8 \mathrm{TeV}$ with the novel CMS technique of data scouting, Phys. Rev. Lett. 117 (2016) 031802

[arXiv: 1604.08907] [INSPIRE].

[24] CMS collaboration, Search for dijet resonances in proton-proton collisions at $\sqrt{s}=13 \mathrm{TeV}$ and constraints on dark matter and other models, Phys. Lett. B 769 (2017) 520 [Erratum ibid. B 772 (2017) 882] [arXiv: 1611.03568] [INSPIRE].

[25] S. Schramm on behalf of the ATLAS collaboration, Search for dark matter in pp collisions at $A T L A S$, PoS (ICHEP2016) 138 [INSPIRE].

[26] A. De Simone and T. Jacques, Simplified models vs. effective field theory approaches in dark matter searches, Eur. Phys. J. C 76 (2016) 367 [arXiv: 1603.08002] [INSPIRE].

[27] A. Berlin, D. Hooper and S.D. McDermott, Simplified Dark Matter Models for the Galactic Center Gamma-Ray Excess, Phys. Rev. D 89 (2014) 115022 [arXiv:1404.0022] [InSPIRE].

[28] M. Cirelli et al., PPPC 4 DM ID: A Poor Particle Physicist Cookbook for Dark Matter Indirect Detection, JCAP 03 (2011) 051 [Erratum ibid. 10 (2012) E01] [arXiv:1012.4515] [INSPIRE].

[29] G. Elor, N.L. Rodd and T.R. Slatyer, Multistep cascade annihilations of dark matter and the Galactic Center excess, Phys. Rev. D 91 (2015) 103531 [arXiv:1503.01773] [INSPIRE].

[30] T. Li, Simplified dark matter models in the light of AMS-02 antiproton data, JHEP 04 (2017) 112 [arXiv: 1612.09501] [INSPIRE].

[31] A.W. Strong, I.V. Moskalenko and V.S. Ptuskin, Cosmic-ray propagation and interactions in the Galaxy, Ann. Rev. Nucl. Part. Sci. 57 (2007) 285 [astro-ph/0701517] [InSPIRE].

[32] V.L. Ginzburg, V.A. Dogiel, V.S. Berezinsky, S.V. Bulanov and V.S. Ptuskin, Astrophysics of cosmic rays, North-Holland, Amsterdam, The Netherlands, (1990).

[33] Q. Yuan, S.-J. Lin, K. Fang and X.-J. Bi, Propagation of cosmic rays in the AMS-02 era, Phys. Rev. D 95 (2017) 083007 [arXiv:1701.06149] [InSPIRE].

[34] S.-J. Lin, Q. Yuan and X.-J. Bi, Quantitative study of the AMS-02 electron/positron spectra: Implications for pulsars and dark matter properties, Phys. Rev. D 91 (2015) 063508 [arXiv: 1409.6248] [INSPIRE].

[35] A. Cuoco, M. Krämer and M. Korsmeier, Novel Dark Matter Constraints from Antiprotons in Light of AMS-02, Phys. Rev. Lett. 118 (2017) 191102 [arXiv:1610.03071] [InSPIRE].

[36] M.-Y. Cui, Q. Yuan, Y.-L.S. Tsai and Y.-Z. Fan, Possible dark matter annihilation signal in the AMS-02 antiproton data, Phys. Rev. Lett. 118 (2017) 191101 [arXiv:1610.03840] [INSPIRE].

[37] J. Feng, N. Tomassetti and A. Oliva, Bayesian analysis of spatial-dependent cosmic-ray propagation: astrophysical background of antiprotons and positrons, Phys. Rev. D 94 (2016) 123007 [arXiv: 1610.06182] [INSPIRE].

[38] X.-J. Huang, C.-C. Wei, Y.-L. Wu, W.-H. Zhang and Y.-F. Zhou, Antiprotons from dark matter annihilation through light mediators and a possible excess in AMS-02 $\bar{p} / p$ data, Phys. Rev. D 95 (2017) 063021 [arXiv:1611.01983] [InSPIRE]. 
[39] S.-J. Lin, X.-J. Bi, J. Feng, P.-F. Yin and Z.-H. Yu, Systematic study on the cosmic ray antiproton flux, Phys. Rev. D 96 (2017) 123010 [arXiv: 1612.04001] [INSPIRE].

[40] H.-B. Jin, Y.-L. Wu and Y.-F. Zhou, Astrophysical background and dark matter implication based on latest AMS-02 data, arXiv:1701.02213 [INSPIRE].

[41] J.-S. Niu and T. Li, Galactic cosmic-ray model in the light of AMS-02 nuclei data, Phys. Rev. D 97 (2018) 023015 [arXiv: 1705.11089] [INSPIRE].

[42] J.F. Navarro, C.S. Frenk and S.D.M. White, The structure of cold dark matter halos, Astrophys. J. 462 (1996) 563 [astro-ph/9508025] [INSPIRE].

[43] J.F. Navarro, C.S. Frenk and S.D.M. White, A universal density profile from hierarchical clustering, Astrophys. J. 490 (1997) 493 [astro-ph/9611107] [INSPIRE].

[44] V.S. Ptuskin, I.V. Moskalenko, F.C. Jones, A.W. Strong and V.N. Zirakashvili, Dissipation of magnetohydrodynamic waves on energetic particles: impact on interstellar turbulence and cosmic ray transport, Astrophys. J. 642 (2006) 902 [astro-ph/0510335] [INSPIRE].

[45] A.W. Strong and I.V. Moskalenko, Propagation of cosmic-ray nucleons in the galaxy, Astrophys. J. 509 (1998) 212 [astro-ph/9807150] [INSPIRE].

[46] I.V. Moskalenko, A.W. Strong, J.F. Ormes and M.S. Potgieter, Secondary anti-protons and propagation of cosmic rays in the galaxy and heliosphere, Astrophys. J. 565 (2002) 280 [astro-ph/0106567] [INSPIRE].

[47] A.W. Strong and I.V. Moskalenko, Models for galactic cosmic ray propagation, Adv. Space Res. 27 (2001) 717 [astro-ph/0101068] [INSPIRE].

[48] I.V. Moskalenko, A.W. Strong, S.G. Mashnik and J.F. Ormes, Challenging cosmic ray propagation with antiprotons. Evidence for a fresh nuclei component?, Astrophys. J. $\mathbf{5 8 6}$ (2003) 1050 [astro-ph/0210480] [INSPIRE].

[49] C. Balázs and T. Li, AMS-02 fits Dark Matter, JHEP 05 (2016) 033 [arXiv:1509.02219] [INSPIRE].

[50] R. Trotta, G. Johannesson, I.V. Moskalenko, T.A. Porter, R.R. de Austri and A.W. Strong, Constraints on cosmic-ray propagation models from a global Bayesian analysis, Astrophys. J. 729 (2011) 106 [arXiv: 1011.0037] [INSPIRE].

[51] K. Auchettl and C. Balázs, Extracting the size of the cosmic electron-positron anomaly, Astrophys. J. 749 (2012) 184 [arXiv:1106.4138] [INSPIRE].

[52] G. Giesen et al., AMS-02 antiprotons, at last! Secondary astrophysical component and immediate implications for Dark Matter, JCAP 09 (2015) 023 [arXiv: 1504.04276] [INSPIRE].

[53] K. Kohri, K. Ioka, Y. Fujita and R. Yamazaki, Can we explain AMS-02 antiproton and positron excesses simultaneously by nearby supernovae without pulsars or dark matter?, PTEP 2016 (2016) 021E01 [arXiv: 1505.01236] [INSPIRE].

[54] B.-Q. Lu and H.-S. Zong, Limits on dark matter from AMS-02 antiproton and positron fraction data, Phys. Rev. D 93 (2016) 103517 [arXiv:1510.04032] [INSPIRE].

[55] W. Liu, X.-J. Bi, S.-J. Lin, B.-B. Wang and P.-F. Yin, Excesses of Cosmic Ray Spectra from A Single Nearby Source, Phys. Rev. D 96 (2017) 023006 [arXiv: 1611.09118] [InSPIRE].

[56] R. Kappl, A. Reinert and M.W. Winkler, AMS-02 Antiprotons Reloaded, JCAP 10 (2015) 034 [arXiv: 1506.04145] [INSPIRE]. 
[57] F. James and M. Roos, Minuit: A System for Function Minimization and Analysis of the Parameter Errors and Correlations, Comput. Phys. Commun. 10 (1975) 343 [InSPIRE].

[58] CMS collaboration, Search for dark matter in final states with an energetic jet, or a hadronically decaying $W$ or $Z$ boson using $12.9 \mathrm{fb}^{-1}$ of data at $\sqrt{s}=13 \mathrm{TeV}$, CMS-PAS-EXO-16-037 [INSPIRE].

[59] G. Steigman, B. Dasgupta and J.F. Beacom, Precise Relic WIMP Abundance and its Impact on Searches for Dark Matter Annihilation, Phys. Rev. D 86 (2012) 023506 [arXiv: 1204.3622] [INSPIRE].

[60] X.-G. He and J. Tandean, New LUX and PandaX-II Results Illuminating the Simplest Higgs-Portal Dark Matter Models, JHEP 12 (2016) 074 [arXiv:1609.03551] [INSPIRE].

[61] S. Baek, P. Ko, W.-I. Park and Y. Tang, Indirect and direct signatures of Higgs portal decaying vector dark matter for positron excess in cosmic rays, JCAP 06 (2014) 046 [arXiv: 1402.2115] [INSPIRE].

[62] G. Arcadi et al., The Waning of the WIMP? A Review of Models, Searches and Constraints, arXiv:1703.07364 [INSPIRE]. 